\title{
Spectral mode-beat phenomena in a cylindrical microcavity
}

\author{
D.J.W. Klunder, M.L.M. Balistreri*, F.C. Blom ${ }^{+}$, A. Driessen, H.J.W.M. Hoekstra, L. Kuipers* and N.F.van Hulst* \\ Lightwave Device Group, Applied Optics Group* \\ MESA $^{+}$Research Institute, University of Twente, P.O. Box 217, \\ 7500 AE Enschede, The Netherlands, e-mail: D.Klunder@el.utwente.nl
}

\begin{abstract}
Detailed spectral analysis of photon scanning tunneling microscope images has been carried out. The analysis of spectral mode-beat phenomena leads to an accurate determination of modeprofiles and gives evidence of counterpropagating modes.
\end{abstract}

\section{INTRODUCTION}

Recently, the Whispering Gallery Modes (WGMs) of a cylindrical integrated optics microcavity (MC) have been mapped with a photon scanning tunneling microscope (PSTM) [1] with unprecedented high spatial and spectral resolution [2]. From these measurements not only the Free Spectral Range (FSR) of the WGMs could be obtained [3], but also spatial and spectral mode-beat have been observed [4]. Both polarization conversion and counterpropagation could be explained from analyzing the spatial mode-beat [4]. In this paper spectral mode-beat phenomena will be discussed. The inclusion of spectral mode-beat allows for resolving the WGMs spectrally and gives a deeper understanding of the propagation characteristics.

\section{WHISPERING GALLERY MODES IN A MICROCAVITY}

The field in a microcavity probed and excited by a straight waveguide (see Figure 1) can be expressed as a geometric sum over the WGMs.. WGMs can be described by the polarization state, slab order $\mathrm{k}$, radial order $l$ and the angular propagation constant [4], [5].
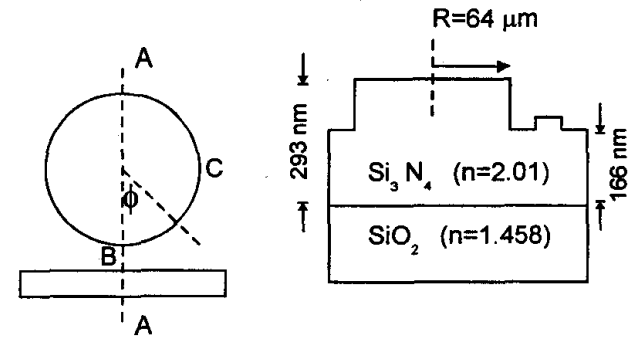

Figure 1 Top-view and cross-section $(A-A)$ of the cylindrical microcavity with adjacent waveguide

The microcavity under consideration has a radius ( $R$ ) of $64 \mu \mathrm{m}$ and is realized in a $\mathrm{Si}_{3} \mathrm{~N}_{4} / \mathrm{SiO}_{2}$ layer-system on a Si substrate [6], [7].

\section{SPECTRAL MODE-BEAT}

From analyzing the field in a microcavity at a fixed radial position as a function of wavelength, an expression for the spectral mode beat period between two modes $(a, b)$ with common origin (point $B$ in Figure 1) can be found:

$$
\Delta \lambda_{\text {beat }}=\frac{2 \pi}{\mid \frac{1}{\mathrm{FSR}_{\mathrm{a}}}-\frac{1}{\mathrm{FSR}_{\mathrm{b}} \mid} \phi}
$$

From Eq. (1) follows that the spectral mode-beat period is not only a function of the FSRs of the modes, but also of the angle of observation in the microcavity (ф).

\section{COUNTERPROPAGATION}

Consider the wavelength-scan for a fixed position in the $\mathrm{MC}$, point $\mathrm{C}$ in Figure 1, and the corresponding Fourier spectrum in Figure 2.
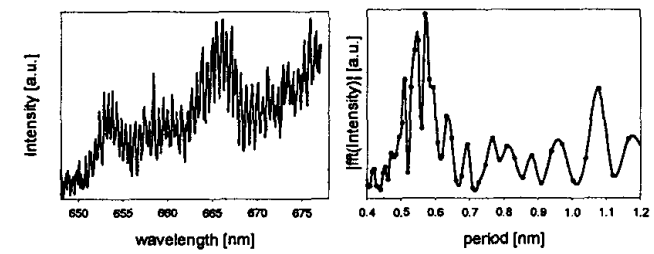

Figure 2 Wavelength scan $(30 \mathrm{~nm})$ at an angle $\phi=90^{\circ}$ and its Fourier spectrum (TE polarized light coupled in)

Besides the peaks with periods around $0.5 \mathrm{~nm}$, which correspond with the FSRs of the TE and TM polarized WGMs, a peak with a period around $1.1 \mathrm{~nm}$ has been observed. This peak is attributed to the spectral modebeat between counterpropagating modes $\left(\Delta \lambda_{\text {beat }}=1.02\right.$ $1.09 \mathrm{~nm})$.

\section{MODEPROFLES}

By considering a wavelength scan at a fixed angle $\phi$

\footnotetext{
${ }^{+}$currently with Uniphase Netherlands B.V., Eindhoven, The Netherlands
} 
and variable radial position, the Fourier spectrum as function of the radial position can be calculated. Taking a fixed period corresponding with the FSR of a mode, it is possible to extract its modeprofile. The intensity profiles resulting from the spectral mode-beat between two modes can be obtained analogously, just by fixing the corresponding period.

In order to resolve the modes of different radial order a scan range larger than $100 \mathrm{~nm}$ is needed, which is not available with the set-up used. As an alternative, we analyzed a wavelength-averaged radial intensity profile as given in Figure 3A. The resulting intensity profile is not only a superposition of the modeprofiles, but also contains a contribution from the spectral mode-beat.

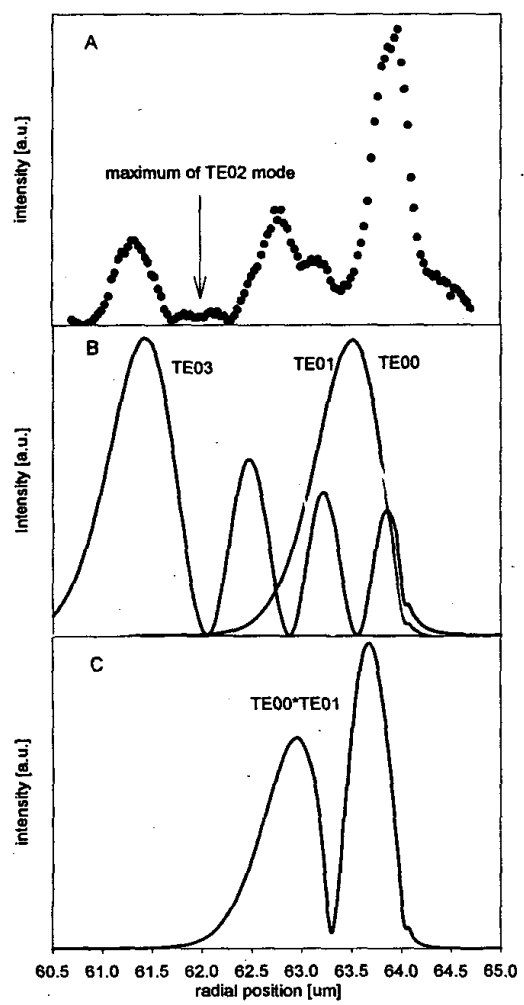

Figure 3 Spectral analysis of $12 \mathrm{~nm}$ wavelength-scan with TE polarized light coupled in: A: wavelength-averaged radial intensity profile. $B$ : calculated modeprofiles for $T E 0(l=0,1,3)$ modes. $C$ : calculated radial intensity profile of TE00-TE01 spectral mode-beat.

From comparing the wavelength-averaged intensity profile (Figure $3 \mathrm{~A}$ ) with the calculated modeprofiles (Figure $3 \mathrm{~B}$ ) it can be concluded that the calculated maxima of the TE03 and TE01 modes agree well with the wavelength-averaged intensity profile. Moreover it can be concluded, from comparing Figure $3 \mathrm{~A}$ with Figure 3B, that the TE02 mode has not been excited. The calculated maximum of the TE00 mode is somewhat to the left of the corresponding maximum in the wavelength-averaged intensity profile. Combining the radial intensity profile of the TE00-TE01 spectral mode-beat (Figure $3 \mathrm{C}$ ) with the modeprofile of the TE00 mode results in a peak shifted to the right, which is in much better agreement with Figure 3A.

\section{CONCLUSIONS}

Counterpropagation of WGMs has been shown spectrally. It is shown that both the modeprofiles and spectral mode-beat intensity profiles can be extracted. The inclusion of spectral mode-beat phenomena in the analysis of PSTM measurements of MCs enables an accurate analysis of the intensity distribution and the propagation characteristics. The spectral analysis is consistent with the results of the spatial analysis [4]

\section{ACKNOWLEDGEMENTS}

This work is supported by the Dutch Foundation for Fundamental Research on Matter (FOM).

\section{REFERENCES}

[1] N.F. van Hulst, M.H.P. Moers, O.F.J. Noordman, R.G. Tack, F.B. Segerink and B. Bölger, "Near-field optical microscope using a silicon-nitride probe", Appl. Phys. Lett., vol. 62, pp. 461-463, 1993. [2] M.L.M. Balistreri, J. Korterik, D.J.W. Klunder, F.C. Blom, A Driessen, L. Kuipers, N.F van Hulst, "Imaging of the Whispering Gallery Modes in cylindrical integrated optical microcavities", in Proc. IEEE LEOS Benelux Chapter, pp. 37-40, November 261998. [3] M.L.M. Balistreri, A. Driessen, D.J.W. Klunder, F.C. Blom, L. Kuipers, H.J.W.M. Hoekstra, N.F. van Hulst, "Imaging of an integrated optics microcavity with a PSTM as function of position and wavelength", IEEE LEOS' '98, PD 2.4, 1998.

[4] D.J.W. Klunder, M.L.M. Balistreri, F.C. Blom, A. Driessen, L. Kuipers, H.J.W.M. Hoekstra, N.F. van Hulst, T.J.A. Popma, “ Analysis of nanometer resolution images of whispering gallery modes in an integrated optic cylindrical micro-resonator", ECIO '99, pp. 113-116, 1999.

[5] Päivi Heimela, Pekka Katila, Jaakko Aarnio and Arja Heinämäki, "Thermally Tunable Integrated Optical Ring Resonator with Poly-Si Thermistor", Journal of Lightwave Technology, vol 14, pp. $2260-2267,1996$

[6] F.C. Blom, D.R. van Dijk, H.J.W.M. Hoekstra, A. Driessen and T.J.A. Popma, "Experimental study of integrated optics microcavity resonators: towards and all-optical switching device", Appl. Phys. Lett., vol. 71, pp. 747-749, 1997.

[7] F.C. Blom, H. Kelderman, H.J.W.M. Hoekstra, A. Driessen, T.J.A. Popma, S.T. Chu and B.E. Little, "A single channel dropping filter based on a cylindrical micro-resonator", Opt. Comm., in press. 